\title{
Observational constraints on annihilation sites in 1E 1740.7-2942 and Nova Muscae
}

\author{
Igor V. Moskalenko* and Elisabeth Jourdain ${ }^{\dagger}$ \\ *Max-Planck-Institut für extraterrestrische Physik, D-85740 Garching, Germany \\ ${ }^{\dagger}$ Centre d'Etude Spatiale des Rayonnements, 31028 Toulouse Cedex, France
}

\begin{abstract}
The region of the Galactic center contains several sources which demonstrate their activity at various wavelengths and particularly above several hundred keV [1]. Escape of positrons from such a source or several sources into the interstellar medium, where they slow down and annihilate, can account for the $511 \mathrm{keV}$ narrow line observed from this direction. 1E 1740.7-2942 object has been proposed as the most likely candidate to be responsible for this variable source of positrons [2]. Besides, Nova Muscae shows a spectrum which is consistent with Comptonization by a thermal plasma $k T_{e} \lesssim 100 \mathrm{keV}$ in its hard X-ray part, while a relatively narrow annihilation line observed by SIGMA on Jan. 20-21, 1991 implies that positrons annihilate in a much colder medium [3,4].

We estimate the electron number density and the size of the emitting regions suggesting that annihilation features observed by SIGMA from Nova Muscae and $1 \mathrm{E} 1740.7-2942$ are due to the positron slowing down and annihilation in thermal plasma. We show that in the case of Nova Muscae the observed radiation is coming from a pair plasma stream $\left(n_{e^{+}} \approx n_{e^{-}}\right)$rather than from a gas cloud. We argue that two models are probably relevant to the $1 \mathrm{E}$ source: annihilation in (hydrogen) plasma $n_{e^{+}} \lesssim n_{e^{-}}$at rest, and annihilation in the pair plasma stream, which involves matter from the source environment.
\end{abstract}

\section{OBSERVATIONS}

Observations with the SIGMA telescope have revealed annihilation features in the vicinity of $\sim 500 \mathrm{keV}$ in spectra of two Galactic black hole candidates (Fig. 1): 1E 1740.7-2942 (1E 1740) and Nova Muscae (NM). Three times a broad excess in the 200-500 keV region was observed in the 1E 1740 emission spectra [6-9]. The features detected on Oct. 13-14, 1990 and Sept. 19-20, 1992 showed similar fluxes and line widths, the lifetime was restricted by 1-3 days. In Oct. 1991 an excess at high energies was observed during 19 days and was not so intensive as two others: the average flux was $(1.9 \pm 0.6) \times 10^{-3}$ phot $\mathrm{cm}^{-2}$ 

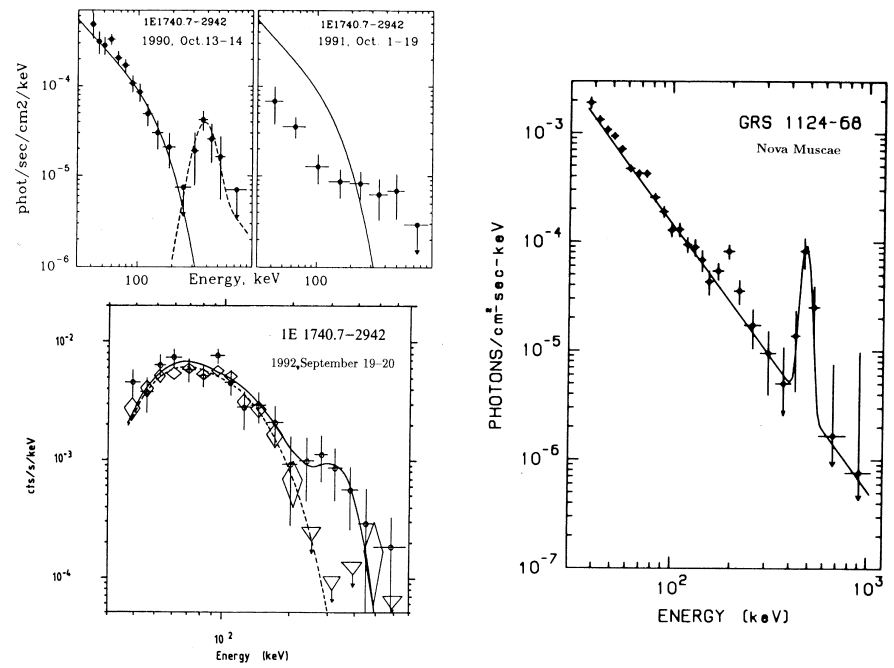

FIGURE 1. Energy spectra of 1E $1740[1,6-9]$ and NM [4] observed by SIGMA are shown together with fits of the authors. For Sept. 1992 flare shown is counts $\mathrm{s}^{-1}$ $\mathrm{keV}^{-1}$. The dashed line in the upper left panel shows the annihilation line shape for Gaussian-like injection of energetic particles into the thermal plasma of $k T=35 \mathrm{keV}$ for $E / A=20$. The line is shifted left to approach the data.

$\mathrm{s}^{-1}$ in the 300-600 keV region. On Jan. 20-21, 1991, the NM spectrum showed a clear emission feature near $500 \mathrm{keV}$ with the intrinsic line width $\lesssim 58 \mathrm{keV}$ $[4,5]$. Meanwhile, during all periods of observation the hard X-ray emission, $\lesssim 200 \mathrm{keV}$, was found to be consistent with the same law. Observations of NM after the X-ray flare are well fitted by a power-law of index $2.4-2.5$, the spectrum of $1 \mathrm{E} 1740$ is well described by Sunyaev-Titarchuk model [10] with $k T \approx 35-60 \mathrm{keV}, \tau \approx 1.1-1.9$. The observational data [4-8] are summarized in the first part of Table 1.

\section{ANALYSIS AND DISCUSSION}

The spectral features observed by SIGMA are, commonly believed, related to $e^{+} e^{-}$-annihilation. Relatively small line widths imply that the temperature of the emitting region is quite low, $k T \approx 35-45 \mathrm{keV}$ for $1 \mathrm{E} 1740$ and $4-5$ $\mathrm{keV}$ for NM. Since the hard X-ray spectra showed no changes, most probably that $e^{+} e^{-}$-pairs produced somewhere close to the central object were injected into surrounding space where they cool and annihilate. Radiation pressure of a near-Eddington source alone can accelerate $e^{+} e^{-}$-plasma up to the bulk Lorentz factor of $\gamma_{0} \sim 2-5$ [11], while Comptonization by the emergent radiation field could provide a mechanism for cooling the plasma which further annihilate 'in flight' (for a discussion see [3]). If there is enough matter around a source, then particles slow down due to Coulomb collisions and annihilate in the medium. We explore further this last possibility by checking whether the inferred parameters of the emitting region are consistent with those obtained by other ways (for details see [12]). We assume single and short particle ejection on a timescale of hours; since the ejection would probably impact on the whole spectrum, longer spectral changes would be observable. 

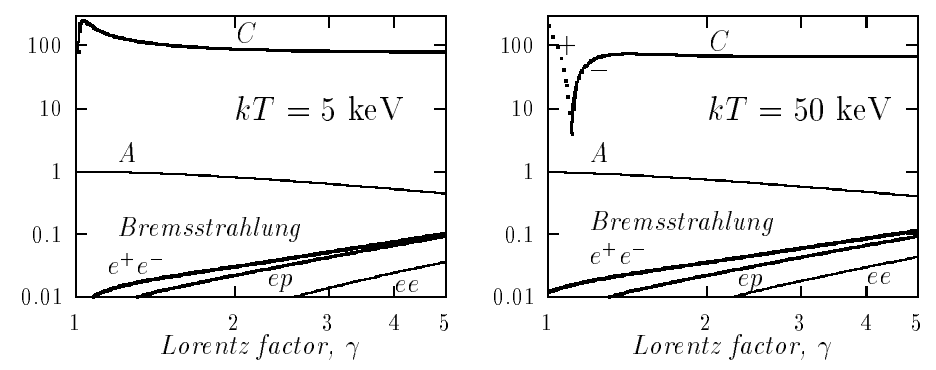

FIGURE 2. The dimensionless annihilation rate (A), and energy losses due to bremsstrahlung and Coulomb scattering $(\mathrm{C})$ in hydrogen plasmas [12].

The relevant energy loss rates $\left|\frac{d \gamma}{d t}\right|$ and annihilation rate per one positron are shown in Fig. 2 in units $n_{e} \pi r_{e}^{2}$. Annihilation rate is small in comparison with the relaxation rate, thus most of positrons annihilate after their distribution approaches the steady-state one.

Suggesting that the energetic particles slow down due to Coulomb scattering in the surrounding matter, one can estimate its (electron) number density $n_{-} \approx \frac{\gamma_{0}-1}{\pi r_{e}^{2} c \Delta_{i}}\left|\frac{d \gamma}{d t}\right|^{-1}$, where $\gamma_{0}$ is the initial Lorentz factor of the plasma stream, $c$ is the light speed, and $\Delta_{i}$ is the time scale of the annihilation line appearance. Taking a reasonable value for the bulk Lorentz factor, $\gamma_{0} \approx 3$, one can obtain $n_{-}^{1 \mathrm{E}} \approx 2.2 \times 10^{7} \mathrm{~cm}^{-3}\left(\Delta_{i} / 2 \text { days }\right)^{-1}$, and $n_{-}^{\mathrm{NM}} \approx 1.5 \times 10^{8} \mathrm{~cm}^{-3}\left(\Delta_{i} / 5 \mathrm{hr}\right)^{-1}$.

If the particles were injected into the medium only once, then the annihilation time scale is $\Delta_{d} \approx \frac{1}{\pi r_{e}^{2} c n_{-} A}$. It yields one more estimate of the number density in the emitting region $n_{-} \approx \frac{1}{\pi r_{e}^{2} c \Delta_{d} A} \approx 1.55 \times 10^{9} \mathrm{~cm}^{-3}\left(\Delta_{d} / 1 \text { day }\right)^{-1}$, where we put $A=A(1) \approx 1$ (Fig. 2). Total duration of this state is $\Delta_{d}^{1 \mathrm{E}} \approx 18-70 \mathrm{hr}$, and $\Delta_{d}^{\mathrm{NM}} \leq 10$ days, that gives $n_{-}^{1 \mathrm{E}} \approx(5-20) \times 10^{8}$ $\mathrm{cm}^{-3}$ and $n_{-}^{\mathrm{NM}} \approx 1.5 \times 10^{8} \mathrm{~cm}^{-3}\left(\Delta_{d} / 10 \text { days }\right)^{-1}$, correspondingly. The values obtained restrict the electron number density in the volume where particles slow down and annihilate (Table 1 ).

The time scales $\Delta_{i, d}$ are connected $\Delta_{d} / \Delta_{i}=\frac{1}{A\left(\gamma_{0}-1\right)}\left|\frac{d \gamma}{d t}\right|$, which is supported also by 1992 Sept. 19-20 observation. Therefore, the annihilation rise time on 1990 Oct. $13-14$ should be $\Delta_{i} \approx 1-2 \mathrm{hr}$ for consistency.

The size of the emitting region $\lambda$ can be estimated from a relation $2 n_{+} \lambda^{3} \approx$ $\Delta_{d} L_{500}$ if we assume $n_{+} \leq n_{-}$for the positron number density. It gives $\lambda^{1 \mathrm{E}} \gtrsim 1.34 \times 10^{13} \mathrm{~cm}\left(\Delta_{d} / 1 \text { day }\right)^{2 / 3} \approx(1.1-2.7) \times 10^{13} \mathrm{~cm}$ and $\lambda^{\mathrm{NM}} \gtrsim 1.3 \times 10^{13} \mathrm{~cm}\left(\Delta_{d} / 10 \text { days }\right)^{2 / 3}$, while the obvious upper limits are $\lambda^{1 \mathrm{E}}<c \Delta_{i} \approx 2.2 \times 10^{14} \mathrm{~cm}\left(\Delta_{i} / 2 \mathrm{hr}\right)$ and $\lambda^{\mathrm{NM}} \leq 5 \times 10^{14} \mathrm{~cm}$.

The column density of the medium where particles slow down should exceed the value $N_{\mathrm{H}} \sim \lambda n_{-}$, viz. $N_{\mathrm{H}}^{\mathrm{NM}} \gtrsim 2 \times 10^{21} \mathrm{~cm}^{-2}\left(\Delta_{d} / 10 \text { days }\right)^{-1 / 3}$, and $2.1 \times 10^{22} \mathrm{~cm}^{-2}\left(\Delta_{d} / 1 \text { day }\right)^{-1 / 3} \lesssim N_{\mathrm{H}}^{1 \mathrm{E}}<c \Delta_{i} n_{-} \approx 1.1 \times 10^{23} \mathrm{~cm}^{-2}$. The column density of the gas cloud measured along the line of sight, where 1E 1740 embedded, is high enough $N_{\mathrm{H}} \approx 3 \times 10^{23} \mathrm{~cm}^{-2}[13,14]$. Note that ASCA measurements give the column density to this source of $\approx 8 \times 10^{22} \mathrm{~cm}^{-2}$ [15]. For NM the corresponding value is $N_{\mathrm{H}} \sim 10^{21} \mathrm{~cm}^{-2}$ [16], less or marginally close to the obtained lower limit. If, on contrary, one suggests $n_{+} \ll n_{-}$it yields a 
TABLE 1. Observational data and parameters of the emitting region.

\begin{tabular}{|c|c|c|c|}
\hline & \multicolumn{2}{|c|}{ 1E $1740.7-2942$} & \multirow{2}{*}{ Nova Muscae } \\
\hline & 1990 Oct.13-14 & 1992 Sep. $19-20$ & \\
\hline Annihilation rise time, $\Delta_{i}$ & $\lesssim 2$ days $^{\mathrm{a}}$ & few hours & $\sim 5 \mathrm{hr}$ \\
\hline Annihilation lifetime, $\Delta_{d}$ & $18-70 \mathrm{hr}$ & $27-75 \mathrm{hr}$ & $\lesssim 10$ days \\
\hline Annihil. line flux, $F_{500}\left(\right.$ phot $\left.\mathrm{cm}^{-2} \mathrm{~s}^{-1}\right)$ & $10^{-2}$ & $4.3 \times 10^{-3}$ & $6 \times 10^{-3}$ \\
\hline Total line flux ${ }^{\mathrm{b}}, L_{500}\left(\right.$ photons s $\left.^{-1}\right)$ & $8.6 \times 10^{43}$ & $3.7 \times 10^{43}$ & $7.2 \times 10^{41}$ \\
\hline Line width, $W(\mathrm{keV})$ & 240 & 180 & 40 \\
\hline Column density, $N_{\mathrm{H}}\left(\mathrm{cm}^{-2}\right)$ & \multicolumn{2}{|c|}{$\sim 10^{23}$} & $\sim 10^{21}$ \\
\hline Plasma temperature, $k T_{e}(\mathrm{keV})$ & \multicolumn{2}{|c|}{$35-45$} & $3-4$ \\
\hline Coulomb energy loss rate, $|d \gamma / d t|$ & \multicolumn{2}{|c|}{70} & 100 \\
\hline Annihilation rate, $A$ & \multicolumn{2}{|c|}{1} & 1 \\
\hline Electron number density, $n_{-}\left(\mathrm{cm}^{-3}\right)$ & \multicolumn{2}{|c|}{$(5-20) \times 10^{8}$} & $1.5 \times 10^{8}$ \\
\hline Size of the emitting region, $\lambda(\mathrm{cm})$ & \multicolumn{2}{|c|}{$(1.1-20) \times 10^{13}$} & $(1.3-50) \times 10^{13}$ \\
\hline
\end{tabular}

a Our estimation is 1-2 hr; $\quad{ }^{\mathrm{b}} 1 \mathrm{E} 1740$ : for $8.5 \mathrm{kpc}$ distance; NM: for $1 \mathrm{kpc}$ distance.

condition $N_{\mathrm{H}} \gg 2 \times 10^{21} \mathrm{~cm}^{-2}\left(\Delta_{d} / 10 \text { days }\right)^{-1 / 3}$, which considerably exceeds the measured value.

These estimations imply that the $500 \mathrm{keV}$ emission observed from NM was coming from $e^{+} e^{-}$-plasma jet $\left(n_{+} \approx n_{-}\right)$rather than from particles injected into a gas cloud ${ }^{1}\left(n_{+} \ll n_{-}\right)$, therefore, particles have to annihilate 'in flight' producing a relatively narrow line shifted dependently on the jet orientation. If so, then our estimation of $n_{-}$from annihilation time scale gives the average electron/positron number density in the jet, fixing the total volume as $\lambda^{3} \sim 2 \times$ $10^{39} \mathrm{~cm}^{3}\left(\Delta_{d} / 10 \text { days }\right)^{2}$. The reported $6 \%-7 \%$ redshift $[4,5]$ supports probably the annihilation-in-jet hypothesis, although its statistical significance is small. The large size of the emitting region and a small width of the line, both except the gravitational origin of the redshift.

For the emitting region in 1 E 1740 our estimations give $n_{-} \gtrsim n_{+}$. Two events, Oct. 1990 and Sept. 1992, have shown similar parameters, which are consistent with single particle injection into the thermal (hydrogen) plasma. The redshift of the line $\sim 25 \%$ [6-8] implies that positrons probably annihilate in a plasma stream moving away from the observer, since the size of the emitting region is too large and rules out its gravitational nature. A natural explanation of this controversial picture is that the plasma stream captures matter from the source environment and annihilation occurs in a moving plasma volume. The value of $n_{-}$obtained is then the average electron number density in the jet, $\lambda^{3} \gtrsim 2.4 \times 10^{39} \mathrm{~cm}^{3}\left(\Delta_{d} / 1 \text { day }\right)^{2}$ is its total volume, and the jet length should be of the order of $\sim 0.2 c \Delta_{d} \approx 5.2 \times 10^{14} \mathrm{~cm}\left(\Delta_{d} / 1\right.$ day $)$.

While some part of the pair plasma annihilates near $1 \mathrm{E} 1740$ producing the broad line, the remainder could escape into a molecular cloud, which was found to be associated with this source $[13,14]$. Taking $\sim 10^{5} \mathrm{~cm}^{-3}$ for the average

1) A possibility that NM lies in front of a large gas cloud can not be totally excluded. In this case, particles could be injected into this cloud, away from the observer. 
number density of the cloud one can obtain for the slowing down time scale ${ }^{2}$ $\Delta_{i} \lesssim 1$ year, the same as was obtained in [2]. The size of the turbulent region caused by propagation of a dense jet should also be of the order of $1 \mathrm{ly}$. It agrees well with the length $2-4$ ly $(15-30$ arcsec at $8.5 \mathrm{kpc})$ of a double-sided radio jet symmetrical about $1 \mathrm{E} 1740$ [17].

If the lines from 1E 1740 (Fig. 1) were produced by continuous injection of energetic particles, then observations of the narrow $511 \mathrm{keV}$ line emission from the Galactic center allows to put an upper limit on the particle escape rate into the interstellar medium. Taking $\tau_{0}=1$ year for the positron lifetime in $10^{5} \mathrm{~cm}^{-3}$ dense molecular cloud [2], and suggesting one hard state of $\Delta_{d} \gtrsim 2$ days long per period $\tau_{0}$, one can obtain an escape rate $E / A \approx \frac{F_{511} \tau_{0}}{F_{500} \Delta_{d}} \lesssim$ 20 , where we took $F_{511} \approx 10^{-3}$ phot $\mathrm{cm}^{-2} \mathrm{~s}^{-1}$ for the narrow line intensity [18], and $F_{500}=10^{-2}$ phot $\mathrm{cm}^{-2} \mathrm{~s}^{-1}$ (Table 1). This is consistent with 1990 Oct. 13-14 and 1992 Sept. 19-20 spectra; the dashed line in Fig. 1 shows the annihilation line shape for Gaussian-like particle injection, $\sim \exp \left[-(\gamma-4)^{2}\right]$, into the thermal plasma of $k T=35 \mathrm{keV}$ for $E / A=20$ [12]. The longest hard state (19 days, Oct. 1991) with the average flux of $F_{500} \approx 2 \times 10^{-3}$ phot $\mathrm{cm}^{-2}$ $\mathrm{s}^{-1}$ places the upper limit at almost the same level $E / A \approx 10$.

I.M. is grateful to the SIGMA team of CESR for hospitality and facilities.

\section{REFERENCES}

1. Churazov E., et al., ApJS 92, 381 (1994)

2. Ramaty R., et al., ApJ 392, L63 (1992)

3. Gilfanov M., et al., Soviet Astron. Lett. 17, 437 (1991)

4. Goldwurm A., et al., ApJ 389, L79 (1992)

5. Sunyaev R. A., et al., ApJ 389, L75 (1992)

6. Bouchet L., et al., ApJ 383, L45 (1991)

7. Sunyaev R. A., et al., ApJ 383, L49 (1991)

8. Cordier B., et al., A\&A 275, L1 (1993)

9. Churazov E., et al., ApJ 407, 752 (1993)

10. Sunyaev R. A., Titarchuk L. G., A\&A 86, 121 (1980)

11. Kovner I., A $6 A$ 141, 341 (1984)

12. Moskalenko I. V., Jourdain E., $A \mathscr{E} A$ in press (1997) - astro-ph/9702071

13. Bally J., Leventhal M., Nature 353, 234 (1991)

14. Mirabel I. F., et al., A\&A 251, L43 (1991)

15. Sheth S., et al., ApJ 468, 755 (1996)

16. Greiner J., et al., in Proc. Workshop on Nova Muscae, ed. S. Brandt, Lyngby: Danish Space Research Inst., 1991, p.79

17. Mirabel I. F., et al., Nature 358, 215 (1992)

18. Mahoney W. A., Ling J. C., Wheaton Wm. A., ApJS 92, 387 (1994)

2) The annihilation lifetime $\Delta_{d}$ was obtained for thermal plasma and is not valid for the cold medium where positrons mostly annihilate in the bound (positronium) state. 